\title{
Investigation of Sheltering Effect on Global Solar Radiation Data Measured by Weather Stations
}

\author{
Keh-Chin Chang ${ }^{1}$ and Hung-Hsun Chen $^{2}$ \\ ${ }^{1}$ Professor, Department of Aeronautics and Astronautics, National Cheng Kung University, Tainan, Taiwan \\ ${ }^{2}$ Graduate Student, Department of Aeronautics and Astronautics, National Cheng Kung University, Tainan, Taiwan
}

\begin{abstract}
In instalment of pyranometers at the weather stations for measuring global solar radiation, it often cannot avoid appearance of obstacles in their surroundings. Investigation of sheltering effect on measurement of global solar radiation is performed through introducing the shelter view factor. Evaluation of the shelter view factor is made by means of a fisheye-lens photograph together with the calculation method developed by Steyn (1980). Taitung weather station (TWS) is chosen for the study. The shelter view factor for the pyranometer installed at TWS is estimated to be $11.8 \%$. Measurements of global solar radiation are conducted at a place where is located nearby TSW but with the condition of zero shelter view factor. Comparison of the data measured at these two sites indicates $4 \%-25 \%$ deviations of global solar radiation observed in different months of 2017. It suggests a need of the correction to the sheltering effect in the measuring process of global solar radiation at the weather stations except those who are not subject to surrounding obstacles in the sky dome.
\end{abstract}

\section{Introduction}

Solar radiation data is definitely necessary for the development, design and operation of solar energy application. Instruments for measuring solar radiation are of two basic types, that is, pyranometer (or solarimeter) and pyrheliometer (or actinometer). A pyranometer is an instrument for measuring total hemispherical (global) solar radiation, while a pyrheliometer is an instrument using a collimated detector for measuring solar radiation from a small portion of sky around the sun (i.e., beam radiation, see figure 1) at normal incidence. In Taiwan, Central Weather Station (CWS) is in charge of providing solar radiation data. There are currently 30 meteorological stations under CWS distributed nationwide in Taiwan who measure global solar radiation constantly by using the pyranometer installed on horizontal surface. Figure 1 shows schematically the composition of global solar radiation over the sky dome. Global (total) radiation incident on ground is basically composed of beam and diffuse components.

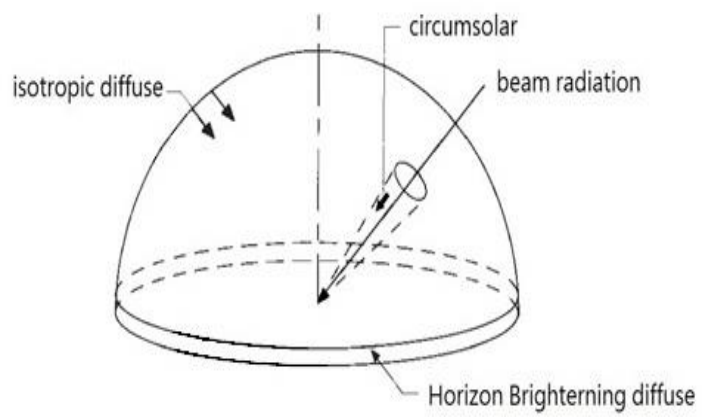

Fig. 1. Schematic of the composition of global radiation over the sky dome. 


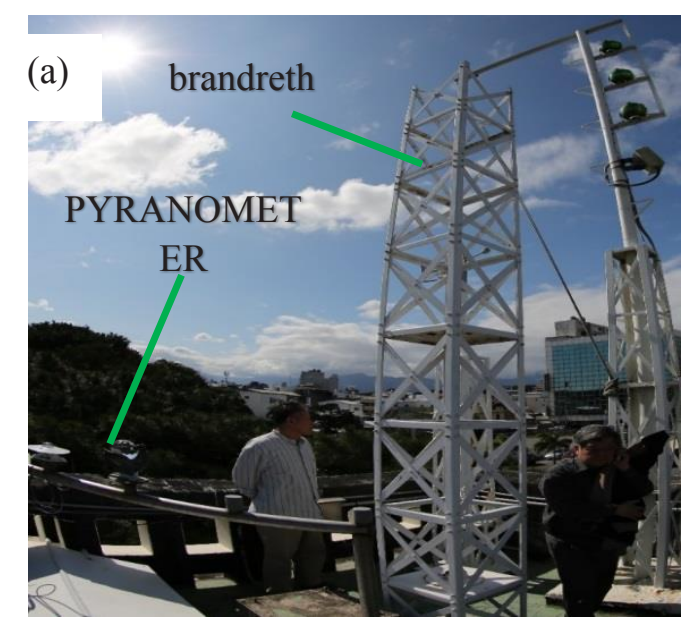

a metal brandreth for placing wind anemometer (height : $4.88 \mathrm{~m}$, distance from pyranometer : $2.37 \mathrm{~m}$ )

(b)

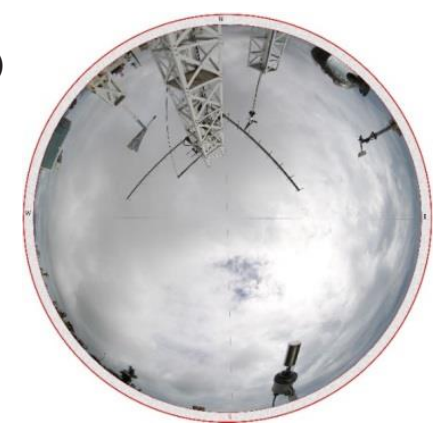

Shelter view factor: $11.8 \%$

Fig. 2. (a) View of the pyranometer and it's surrounding and (b) Fisheye photograph centered nearby the pyranometer on the roof of Taitung weather station.

Beam radiation arrives on the ground directly, without being scattered in the atmosphere, while diffuse radiation is the scattering part due to obstruction by particulates and water droplets in the atmosphere on the route to the ground. Diffuse radiation can be further split into three parts: isotropic, circumsolar, and horizon brightening diffuse parts. The circumsolar diffuse radiation results from forward scattering of solar radiation and is concentrated around the sun (beam) ray. The horizon brightening diffuse radiation results from the refraction process of air and contributes little to total except at around sunrise or sunset time. In a real situation, there might exist some obstacles (shelters) around the installed pyrometer as shown in figure 2a. The shelters may block the beam and circumsolar diffuse radiation but definitely block some isotropic diffuse radiation incident upon the pyrometer. The shelters may also reflect the beam radiation onto the pyrometer. The study aims at (1) evaluating the sheltering extent in terms of view factor and (2) investigating the sheltering effect by comparing the global radiation data measured at a selective weather station with the in-situ data measured at a nearby location but with zero shelter condition.

\section{Testing sites}

Taitung weather station (TWS; $121^{\circ} 08^{\prime} 48^{\prime \prime}$ E, $22^{\circ} 45^{\prime} 15^{\prime \prime}$ $\mathrm{N}$ ), where is located in the east part of Taiwan proper, is selected for the study. The data of solar global radiation, in terms of the typical meteorological year (TMY) between 2004 and 2013, measured at TWS is referred to the recent study of Chang et al. [1]. The in-situ measurements of global radiation under zero shelter condition were carried out at the Taitung Centre of National Open University (TCNOU; $121^{\circ} 07^{\prime} 20^{\prime \prime} \mathrm{E}$, $22^{\circ} 45^{\prime} 19^{\prime \prime} \mathrm{N}$ ), where is located $3.3 \mathrm{~km}$ in distance away from TWS.

\section{Experimental methods}

\subsection{Fisheye images}

The fisheye images were taken with a Canon 5D Mak III digital camera together with a Canon Fisheye USM lens (EF 8-15 mm f/4L). The lens generates a field of view $180^{\circ}$ in a picture such as shown in figure 2(b).

\subsection{Evaluation of view factor from fisheye-lens photograph}

Referring to figure 3 , the conical projection of an obstacle with the surface $A_{j}$ on the sphere is $B_{j}$, and its normal projection on the base plane, i.e. its image on the fisheye picture, is $C_{j}$. According to the definition of solid angle, the solid angle $\left(\Omega_{j}\right)$ constructed by $B_{j}$ is defined to be $B_{j} / r^{2}$, where $\mathrm{r}$ is the radius of the sphere. The view factor is thus calculated as $\Omega_{j} / 2 \pi$, which is equivalent to the fraction of the circle occupied by $C_{j}$, i.e. $=\frac{C_{j}}{\pi r^{2}}, \quad[2]$. Evaluation of view factors due to surrounding shelters (hereafter name as shelter view factor) is stated as follows. First, the fisheye picture was printed with an overlain polar grid such as shown in figure 4. In the print, the obstacles were delineated. With the help of the polar grid the viewing angle of each annulus (36 annular rings were used in the study) are to be counted. After the shelter view factor was calculated for each annulus according to the Steyn method [3], the values were summed up for the total shelter view factor.

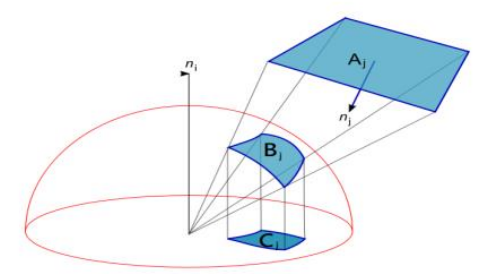

Fig. 3. Fisheye-lens projection $(\mathrm{Cj})$ of an obstacle (Aj) 


\subsection{Global solar radiation measurements}

Measurements of global solar radiation were made by a pyranometer (Model PSP of Eppley Laboratory, Inc.), which is classified as the first standard pyranometer according to ISO 9060: 1990 [4] but has stopped production since 2013, at TWS. In contrast, a pyranometer (Model SMP 11 of Kipp \& Zonen, Inc.), which is classified as the second standard pyranometer [4], were used for measuring global solar radiation in the study at TCNOU. Details of the data acquisition and calculation processes follow the study of Kuo and Chang [5] and are not repeated here. As seen in figure 1, solar radiation incident at solar altitude angles of less than $5^{\circ}$ is of the horizon brightening diffuse component and makes only a very small $(<1 \%)$ contribution to the total. Thus, the solar radiation limited to an elevation of $5^{\circ}$ from the horizon can normally be neglected [6]. Accordingly, the shelter portions located within the solar altitude angles $<$ $5^{\circ}$ were not considered in the calculation of total shelter view factor.

\section{Results and discussion}

The calculated shelter view factor for the pyranometer installed at TWS (figure 1) is $11.8 \%$ in contrast to $0 \%$ shelter view factor for the one installed at TCNOU. As observed from figure 1, the major obstacle is the brandreth (with the height of $4.88 \mathrm{~m}$ ) for placing wind anemometer, which is located in around the north of the pyranometer with the distance of $2.37 \mathrm{~m}$. The sun trajectories on summer (with the highest solar altitude angle) and winter (with the lowest solar altitude angle) solstices are also plotted in figure 4. Clearly, the windanemometer brandreth does not block the beam (in addition to circumsolar diffuse) radiation, but it could cause reflection contribution of the beam solar radiation in few days of a year. However, its contributions to total are insignificant due to the small view factor from the wind-anemometer tower to the pyranometer.

Figure 5 compares the data of global solar radiation (G) of 2017 reported by TWS [7] and those measured in the study. The relative deviation $(\Delta \mathrm{G})$ is calculed by
$\Delta \mathrm{G}=\frac{G_{T C V O U}-G_{T W S}}{G_{T C V O U}} \times 100 \%$

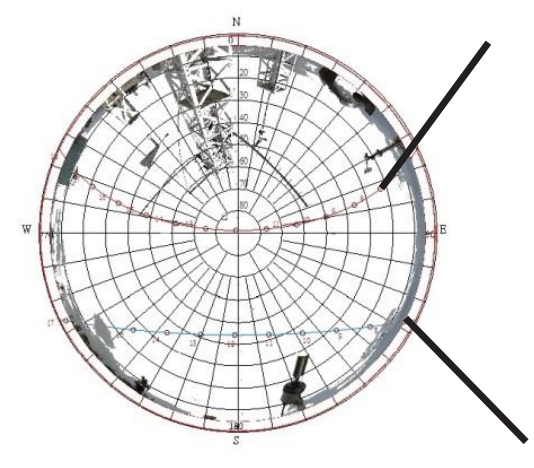

Sun trajectory on summer solstice
Sun trajectory on winter solstice

Fig. 4. Polar grid representation of the fisheye picture taken at Taitung weather station.

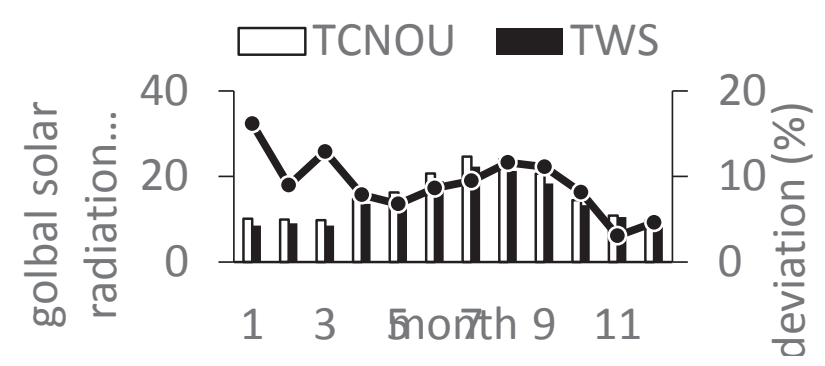

Fig. 5. Comparison of global solar radiation measured at Taitung weather station (TWS, with $11.8 \%$ shelter view factor) and at Taitung Centre of National Open University (TCNOU, with $0 \%$ shelter view factor) in 2017.

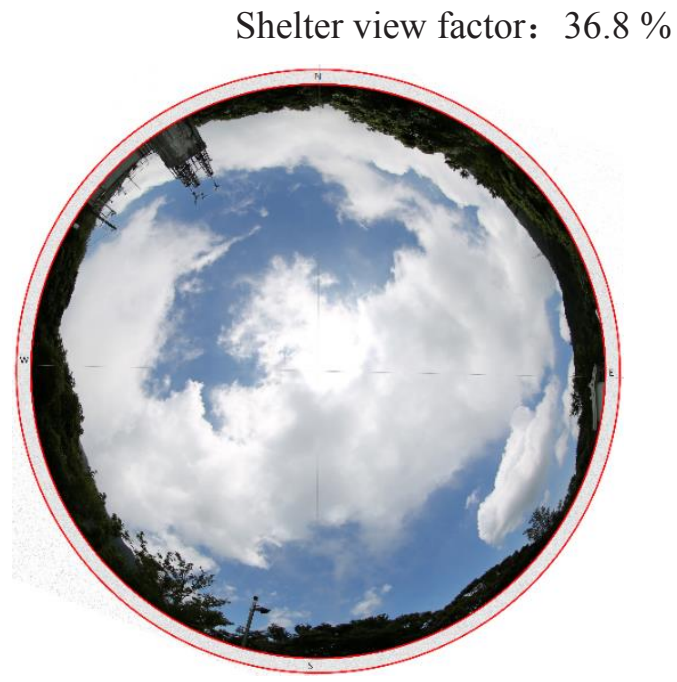

Fig. 6. Fisheye photograph centered nearby the pyranometer on the roof of Anbu weather station.

Since the value of shelter view factor for the pyranometer installed at TCNOU is zero, the data measured there are taken as the basis for comparison. Clearly, the data measured at TSW are lower than those measured at TCNOU (deviations from $4 \%$ to $25 \%$ in different months). As discussed in the preceding section, the primary reduction of solar radiation at TWS is attributed to the isotropic diffusion component due to the sheltering effects of another instruments/facilities installed by the weather station itself (see figure 1(a)). However, taking Anbu weather station, which is located in the mountain area of Yangmingshan National Park, as another example, its fisheye picture (figure 6) exhibits a situation that the sheltering effects are primarily stemmed from the surrounding hills and trees. The calculated view factor for the pyranometer at Anbu weather station is as high as $36.8 \%$. It is expected that the sheltering effect on the measured global solar radiation must be more significant than that at TWS.

\section{Conclusions}

Investigation of sheltering effect on measurement of global solar radiation is performed by means of the shelter view factor around the installed pyranometer. 
Evaluation of the shelter view factor is made by using an fisheye-lens photograph together with the calculation method developed by Steyn [2]. To assess the actual sheltering effect, the global solar radiation reported at TWS (with 11.8\% shelter view factor) is selected for demonstration. A pyranometer is installed at TCNOU who possesses an environment of zero shelter view factor. Comparison of the data between these two measurement sites indicates that $4 \%-25 \%$ deviations are observed in different months of 2017 . It suggests that a correction to the sheltering effect had better be made in the routine measurements of global solar radiation in the weather stations in order to provide more accurate data of global solar radiation.

\section{References}

1. Chang K C, Yen W L and Liu C W 2016 J. Taiwan Energy 3 (1) 89 (in Chinese)

2. Howell J R 1982 A Catalogue of Radiation Configuration Factors (New York: McGraw-Hill)

3. Steyn D G 1980 Atmosphere-Ocean 18 (3) 254

4. ISO 9060: 1990 Solar energy-specification and classification of instruments for measuring hemispherical solar and direct solar radiation http://www.iso.org/standard/16629.html

5. Kuo C W and Chang K C 2015 J. Chinese Institute Engineers 38 (6) 723

6. John H E and Cunningham J R 1983 Physics of Radiology (Hingham, MA: Charles River Media)

7 http://e-service.cwb.gov.tw/HistoryDataQuery/index. jsp 